\title{
Turbulent Mixing and Combustion of
} Supercritical Jets

\author{
Shao Teng Chong, Yihao Tang, Malik Hassanaly and Venkat Raman \\ Department of Aerospace Engineering, University of Michigan, Ann Arbor, MI, 48109, U.S.A
}

\begin{abstract}
Supercritical flows are becoming increasingly relevant to aircraft engines, and have always been integral to rocket motors. More recently, supercritical combustion is being considered for stationary gas turbines in $\mathrm{CO}_{2}$ based cycles. The purpose of this study is to understand the turbulent mixing as the flame structure of fuel/air jets issuing at supercritical conditions. Direct numerical simulations (DNS) of a coflowing $\mathrm{CH}_{4} / \mathrm{O}_{2} / \mathrm{CO}_{2}$ jet with two different inflow configurations are studied at 200 bar pressure - (1) jet with coflow, and (2) jet and annular with coflow. Further, a steady laminar flamelet model is adapted for supercritical conditions and the results of the DNS compared against the flame structure predicted by the flamelet model. It is seen that DNS results are roughly similar to the flamelet results, but behave as a more strained flame as compared to the 1-D results. This suggests that the weak heat release associated with strong dilution broadens the reaction zone, which partially invalidates the 1-D flamelet assumption. In comparing the two inlet configurations, the jet case is shown to have a lower maximum temperature at $\sim 1500 K$ while the annular case has a much higher flame temperature at $\sim 1900 \mathrm{~K}$. The jet case is characterized by an attached flame while the annular case has a highly lifted flame with high strain rate mixing downstream that enhances mixing but forms high temperature, locally fuel rich region that produces an order of magnitude higher CO mass fraction than the jet case. These configurations demonstrate the extreme sensitivity of supercritical flames to inflow conditions. In particular, local hot spots that occur due to inadequate dilution present a design issue.
\end{abstract}

\section{Nomenclature}

$\begin{array}{ll}R e & \text { Reynolds number } \\ P r & \text { Prandtl number } \\ u & \text { Velocity vector } \\ T & \text { Temperature }[\mathrm{K}] \\ \rho & \text { Density }\left[\mathrm{kg} \mathrm{m}{ }^{-3}\right] \\ p & \text { Pressure, }[\mathrm{Pa}] \\ V & \text { Molar volume }\left[\mathrm{m}^{3} \mathrm{~mol}^{-1}\right]\end{array}$

\section{Introduction}

Supercritical mixing and combustion is increasingly important to practical combustors, including highpressure aircraft engines that involve fuel injection at supercritical pressures, rocket combustors that operate at high chamber pressures, and supercritical $\mathrm{CO}_{2}$ cycle for power generation that involves methane or coal combustion in oxygen (instead of air) but with supercritical $\mathrm{CO}_{2}$ as the diluent. While much of the benefit from supercritical mixtures comes due to the increased thermodynamic efficiency associated with high operating pressures in the combustion chambers, such fluids also allow efficient transportation. For instance, the high density and low diffusivity reduces the energy loss in pumping the fluid to the combustor.

Prior studies of supercritical mixing and combustion have mainly focused on rocket combustors, ${ }^{1,2}$ where 
fuel is mixed with oxidizer prior to combustion. It has been observed that the high density ratio of the streams combined with the non-ideal thermodynamic relations lead to changes in mixing behavior. Near to the critical point, supercritical fluids surface tension and enthalpy of vaporization approach zero and specific heat increases exponentially along with density. All these phenomena pose an interesting problem for modeling because of the wildly different extremities in length and time scales that need to be resolved. In direct numerical simulation studies by Bellan and co-workers, ${ }^{3,4}$ several anomalous features including uphill diffusion of species have been noted. These studies focused predominantly on temporal shear layers. One-dimensional studies by Oefelein et al. ${ }^{5}$ have shown significant differences in flame structure at operating pressures close to critical point of the mixture.

The focus of this work is on supercritical $\mathrm{CO}_{2}\left(\mathrm{sCO}_{2}\right)$ cycles for power generation with direct-fired high pressure combustors. According to McClung et al. ${ }^{6}$, supercritical $\mathrm{CO}_{2}$ gas turbine power generation cycles are able to reach cycle thermal efficiency target of $64 \%$ while cutting down on the turbine size and saving at least two heat exchangers because of the direct-firing of methane and oxygen into the supercritical $\mathrm{CO}_{2}$ working fluid. In such cycles, oxy-combustion of fuel (typically methane or pulverized coal) heavily diluted with $\mathrm{CO}_{2}$ (>95\% by mass) is used to provide the energy source. The operating pressures are expected to be around 200 bar or higher. Under such conditions, many different issues arise regarding the combustion process. Preliminary kinetics studies indicate that combustion processes will proceed rapidly at these conditions once mixing is complete. At the same time, presence of local hot spots can lead to device failure if these fluid pockets reach the combustor walls. Hence, the temperature rise inside the combustor is minimized by dilution with $\mathrm{sCO}_{2}$. As will be shown here, at such high dilution levels, the combustion process is rapid such that stable combustion is possible, but is also susceptible to strain-induced extinction. Hence, designing the mixing process is very critical.

In practical combustors, fuel-air mixing and flame structure are of vital importance. In particular, the effect of real gas law on jet evolution, mixing, and subsequent reaction processes needs to be understood in detail. Building on prior DNS studies, the focus here is on two engine-relevant configurations: 1) A coflowing supercritical jet and, 2) an annular configuration with a central fuel jet, an annular oxygen $/ \mathrm{sCO}_{2}$ stream and an outer $\mathrm{sCO}_{2}$ stream. The objective is to determine the turbulence and mixing characteristics in such jets and to study the flame stabilization process.

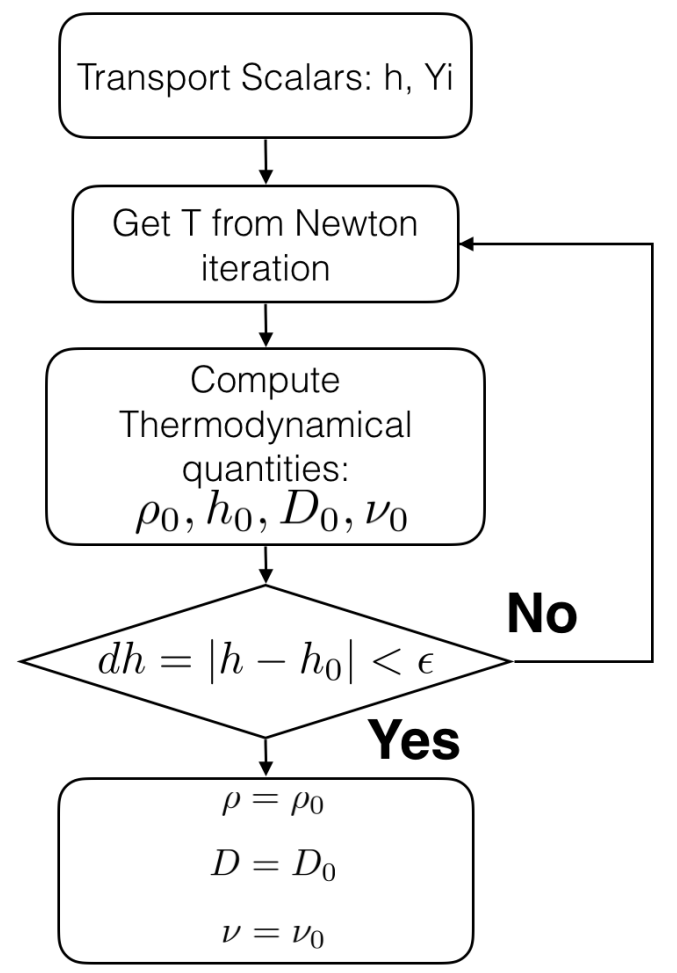

Figure 1. Newton iteration algorithm to determine the thermodynamic properties given the transported scalars 


\section{I.A. Computational formulation for supercritical combustion}

\section{I.A.1. Low-Mach number direct numerical simulation solver for supercritical flows}

The flows considered here, as opposed to rocket combustion, involve very low velocities and are generally in the low-Mach number regime. Consequently, significant computational speed-up can be achieved by formulating the numerical approach to remove acoustic components of the flow. This is typically carried out by the Poisson-equation based pressure correction approach. ${ }^{7,8}$ Here, at each time-step, a Poisson equation is used to enforce mass continuity, which has the physical implication of adjusting the pressure field at infinite speed. In other words, the acoustic waves are assumed to be removed instantaneously from the domain, leading to the equilibrated velocity field at the next time-step. Low-Mach solvers have been widely used for simulating reacting flows, ${ }^{9-12}$ including complex geometries such as model aircraft combustors. ${ }^{13}$ Here, this low-Mach number solver is adapted for supercritical flows by incorporating the real gas thermodynamic properties.

These modifications were implemented in the NGA structured grid solver. ${ }^{8}$ In the simplest implementation of a low-Mach number approach, transport equations for momentum and scalars are first solved to obtain an intermediate solution. A thermochemical density field is then obtained from this solution, which is then used to enforced continuity by adjusting the velocity field. This change to the velocity field is obtained by a Poisson equation solution. The low-Mach number approach followed here is a variation of this basic scheme and is explained in detail elsewhere. ${ }^{8,13}$ In many applications, a conserved or reactive scalar is used to map the gas phase density field. In other words, the density field is obtained directly from an external function.

Here, this approach is not useful for several reasons. Since the gas phase scalars are directly evolved, an a priori relation between scalars and density is not readily available. At the same time, since the real gas equation is more complex and would require at least a 5 dimensional table to populate all the parameters, it is not cost-effective to store such a table and perform interpolation at every step. Furthermore, a refined table to store the large density gradient close to the critical region is not computationally tractable. Finally, when the application spans a region in pressure-density space that is close to the critical point, small changes in temperature can lead to large changes in density as mentioned. Consequently, a direct density feedback can cause the solver to become unstable. This has been observed in other contexts previously, for instance when coupling density to momentum equations in transported probability density function (PDF) methods. ${ }^{9,14,15}$ Here, to overcome these issues and to take into account the departure function in $C_{p}$ value in the diffusion term, a sensible enthalpy equation is transported along with the other governing equations. At each time-step, the source term for enthalpy is computed from the chemistry mechanism via Chemkin modules (described in Sec. II..1). The density field is then obtained by inverting the enthalpy relation to temperature, assuming constant thermodynamic pressure (which is equivalent to the low-Mach assumption).

The low-Mach number formulation is based on the energy-conservative staggered position-time algorithm using a structured, conservative finite-difference scheme. ${ }^{8}$ Time advancement is implemented using the semiimplicit second-order Crank-Nicolson scheme while the scalar transport is using the $2^{\text {nd }}$ order BQUICK (Bounded-QUICK) scheme ${ }^{16}$ to reduce oscillations at lower and upper bounds for critical scalars like species mass fractions used in this study.

The general enthalpy and species mass fraction scalar transport equation is described in the equation below.

$$
\frac{d(\rho \phi)}{d t}+\nabla \cdot(\rho u \phi)=\nabla \cdot(\rho D \nabla \phi)+\rho \dot{\omega}
$$

where $\rho$ is the density, $u$ is the velocity vector, $\phi$ represents the scalar quantities, $D$ is the scalar diffusion coefficient described by the ratio of the thermal conductivity and constant pressure heat capacity for the mixture, $k / C_{p}$, and $\dot{\omega}$ is the source term of the scalars from combustion. Scalar transport diffusivity and viscosity were calculated based on the Chung et al. method ${ }^{17}$ from the Chapman-Enskog theory as explained in section I.A.3. Mixing rules from the corresponding method were also used.

To compute the chemical source terms, the enthalpy and species mass fractions are computed from Chemkin 0-D modules based on initial species mass fraction, temperature, and time-step. Enthalpy change from Chemkin is then corrected with Eq.11 to account for non-ideal effects and departure functions from heat capacity. The updated sensible enthalpy value is then used to calculate the local temperature using Newton iteration method where a user-defined convergence threshold residual (1e-06) is specified. With the 
new temperature, the density in each cell can be backed out from the pressure, temperature and local species mass fractions using a form of Eq.2. The diagram in Fig.1 shows the relationship between the transported scalars, their source terms, and the iterative method used to compute temperature and density.

\section{I.A.2. Real Gas Thermodynamic Properties}

To incorporate real gas effects, the cubic Peng-Robinson equation of state (EOS) will be used in this study. This EOS takes into account the non-linearity between pressure and density at the supercritical and especially the transcritical region where density gradient with respect to changes in temperature is high. This EOS is robust, accurate, and numerically efficient to implement. The pressure-density-temperature relation can be expressed as:

$$
P=\frac{R_{m o l} T}{V_{m}-B_{m}}-\frac{A_{m}}{V_{m}^{2}+2 V_{m} B_{m}-B_{m}^{2}},
$$

where $R_{m o l}$ is the universal molar gas constant and $V_{m}$ the molar volume related to the density as

$$
V_{m}=\frac{M W}{\rho},
$$

where $M W$ is the molecular weight of the fluid. $A_{m}$ and $B_{m}$ are functions of the individual species properties as described below:

$$
\begin{gathered}
A_{i j}=\frac{0.457236 \alpha_{i j} R_{m o l}^{2} T_{c_{i j}}{ }^{2}}{P_{c_{i j}}}, \\
A_{m}=\sum_{i} \sum_{j} X_{i} X_{j} A_{i j} \\
B_{i}=\frac{0.0777961 R_{m o l} T_{c}}{P_{c}}, \\
B_{m}=\sum_{i} X_{i} B_{i} \\
\alpha_{i j}=\left[1+\left(0.37464+1.54226 \omega_{i j}-0.26992 \omega_{i j}{ }^{2}\right)\left(1-\sqrt{\frac{T}{T_{c_{i j}}}}\right)\right]^{2} . \\
\omega_{i j}=\frac{1}{2}\left(\omega_{i i}+\omega_{j j}\right)
\end{gathered}
$$

In the above relations, the acentric factor $\omega$ is a measure of the non-sphericity of the molecules, with this factor being 0.040 for $\mathrm{N}_{2}$ as an example. $T_{c}$ and $P_{c}$ are the critical temperature and pressures of the fluid, which are $126.19 \mathrm{~K}$ and $3.40 \mathrm{MPa}$ for $N_{2}$ as an example. Mixing rules for the application of Peng-Robinson EOS is described in Congiunti et al. ${ }^{18}$ and Harstad et al. ${ }^{19}$ Mixing rules for the other parameters in the equations above can be found in the next section.

In the low-Mach number solver, the effect of changes to the thermochemical state of the mixture affects the flow field predominantly through changes to the density field. In a practical algorithm, this density change at each time-step is supplied to the solver (based on a combustion or mixing model). This density change then adjusts the velocity field through a pressure-Poisson equation. Due to the large density changes associated with the supercritical-to-subcritical pressure changes, and heat release due to chemical reactions, a direct feedback of the density solver will lead to a divergence of the pressure-Poisson equation. Here, an alternative approach is formulated, based on prior experience with transported probability density function (PDF) approach for combustion modeling. ${ }^{14,20}$ In this formulation, a sensible enthalpy equation is transported in the low-Mach number solver, and the density change obtained from changes in enthalpy. In the continuous space-time limit, this approach is identical to the density feedback. However, for finite grids, the use of the transport equation is equivalent to a Lagrangian filter that smooths the density change spatially. The sensible enthalpy equation is given by: 


$$
\rho \frac{D h}{D t}=\frac{1}{\operatorname{RePr}} \frac{k}{C_{p}} \nabla^{2} h+S_{h},
$$

where $S_{h}$ is the chemical source term related enthalpy change. This enthalpy is related to the EOS as folows:

$$
h=G-T\left(\frac{d G}{d T}\right)_{p, X}=h^{0}+p v-R T+K_{1}\left(A_{m}-T \frac{d A_{m}}{d T}\right),
$$

where $G$ is the Gibbs energy, $h^{0}$ denotes the low-pressure reference enthalpy that can be calculated from NASA thermodynamic data coefficients for each species, and $K_{1}$ and $\frac{d A_{m}}{d T}$ are described as

$$
K_{1}=\frac{1}{2 \sqrt{2} B_{m}} \ln \left(\frac{V_{m}+(1-\sqrt{2}) B_{m}}{V_{m}+(1+\sqrt{2}) B_{m}}\right),
$$

and

$$
\frac{d A_{m}}{d T}=\frac{0.457236 R^{2} T_{c}^{2}}{P_{c}}\left(\frac{k^{2}}{T_{c}}-\frac{k(1+k)}{\sqrt{T T_{c}}}\right)
$$

\section{I.A.3. Real Gas Transport Properties}

Viscosity, thermal conductivity and mixing rules were computed using the Chung et al. ${ }^{17}$ method which is based on the Chapman-Enskog theory. It is selected for its accuracy near the critical point and numerical tractability compared to other corresponding state theory-based methods. Readers are referred to Congiunti et al. ${ }^{18}$ and Chung et al. ${ }^{17}$ for the rigorous calculation of thermodynamic parameters specified in this section. For sake of brevity, only the main idea and equations for calculating viscosity and thermal conductivity are laid out in this section.

For supercritical fluid, the Chung et al. viscosity has a correction term, $\eta^{*}$ to the low pressure viscosity term, $\eta^{0}$, and the complete formulation is given by: $[\mathrm{Pa} \cdot \mathrm{s}]$

$$
\begin{gathered}
\eta=\eta^{*} \eta^{0}=1.0 \times 10^{-7}\left(\frac{1}{G_{2}}+E_{6} y+\frac{\Omega_{v}}{F_{C m} \sqrt{T_{m}{ }^{*}}} \eta^{* *}\right)\left(\frac{40.785 F_{C m} \sqrt{M_{m} T}}{V_{C_{m}}^{\frac{2}{3}} \Omega_{v}}\right) \\
F_{C m}=1-0.2756 \omega_{m}+0.059035 \mu_{r m}^{4}+k_{m}
\end{gathered}
$$

where $M_{m}$ is the mixture molecular weight, $T$ is the temperature, $V_{C_{m}}$ is the mixture critical volume, $\Omega_{v}$ is the collision integral as a function of temperature, $F_{C_{m}}$ is an empirical factor depending on mixture acentric factor, $\omega_{m}$, dimensionless dipole moment, $\mu_{r_{m}}$, and a correction factor for polar substances, $k_{m}$, as described in equation 15. $\eta^{* *}, G_{2}$ and $E_{6}$ are computed from Chung et al parameters described in Congiunti et al. ${ }^{18}$ as a function of $\omega_{m}, \mu_{r_{m}}$, and $k_{m} . T_{m}$ and $y$ are computed from equations below.

$$
\begin{gathered}
T_{m}=1.2593 T_{r} \\
y=\frac{\rho_{m} V_{C_{m}}}{6}
\end{gathered}
$$

where $T_{r}$ is the reduced temperature and $\rho_{m}$ is the molecular density.

Similarly, the thermal conductivity is computed using Chung et al. method as shown in Eq 18 below.

$$
\lambda=\frac{31.2 \eta^{0} \Psi}{M_{m}}\left(G_{2}^{*-1}+B_{6} y\right)+q B_{7} y^{2} \sqrt{T_{r}} G_{2}{ }^{*}
$$

where $\eta^{0}$ is the low pressure viscosity term, $\Psi, G_{2}{ }^{*}, B_{6}$ and $B_{7}$ are parameters calculated using the same procedure as in the viscosity calculations. $q$ is defined as in Eq. 19 below:

$$
q=3.586 \times 10^{-3} \frac{\sqrt{\frac{T_{C_{m}}}{M_{m}}}}{V_{C_{m}}{ }^{\frac{2}{3}}}
$$

where $T_{C_{m}}$ is the mixture critical temperature. 
In terms of mixing rules for calculating thermodynamic and transport properties for mixtures, the following mixing rule is used according to Reid et al. ${ }^{21}$ To determine the mixture critical temperature, $T_{C_{m}}$ and volume, $V_{C_{m}}$ :

$$
\begin{gathered}
T_{C_{m}}=\frac{1}{V_{C_{m}}{ }^{\frac{1}{4}}} \sum_{i} \sum_{j} x_{i} x_{j} V_{C_{i j}}{ }^{\frac{1}{4}} T_{C_{i j}} \\
V_{C_{m}}=\sum_{i} \sum_{j} x_{i} x_{j} V_{C_{i j}} \\
T_{C_{i j}}=\sqrt{T_{C_{i}} T_{C_{j}}} k_{i j} \\
V_{C_{i j}}=\frac{1}{8}\left(V_{C_{i}}{ }^{\frac{1}{3}}+V_{C_{j}}{ }^{\frac{1}{3}}\right)^{3} \\
P_{C_{i j}}=Z_{c, i j} R_{m o l} \frac{T_{C_{i j}}}{V_{C_{i j}}} \\
Z_{c, i j}=\frac{1}{2}\left(Z_{c, i i}+Z_{c, j j}\right)
\end{gathered}
$$

where $k_{i j}$ are empirically determined binary interaction parameters for each species pair in this study while $T_{C_{i j}}, V_{C_{i j}}$, and $P_{C_{i j}}$ are binary critical temperature, volume and pressure. $Z_{c, i j}$ is the binary compressibility factor with $Z_{c}$ for each species $i$ and $j$ obtained empirically.

\section{Numerical Method}

Direct numerical simulations (DNS) of two cylindrical jet configurations were carried out: (1) Premixed coflow of oxidizer with $\mathrm{CO}_{2}$ diluent, (2) annular $\mathrm{O}_{2} / \mathrm{CO}_{2}$ case with coflowing $\mathrm{CO}_{2}$ diluent . Both cases are running at conditions and stoichiometry pertinent to a supercritical gas-turbine combustor inlet.

The simulations were run using an in-house code developed in the University of Michigan. Real-gas effects explained above were implemented into a low-Mach number code. The low-Mach number formulation is based on the energy-conservative staggered position-time algorithm using a structured, conservative finitedifference scheme. ${ }^{8}$ Time advancement is implemented using the semi-implicit second-order Crank-Nicolson scheme while the scalar transport is using the 2nd order BQUICK (Bounded-QUICK) scheme to reduce oscillations at lower and upper bounds for critical scalars like species mass fractions used in this study, as explained in the previous section. The real gas thermodynamic and transport properties explained in the previous section were implemented in the existing solver.

This DNS simulation transports a sensible-enthalpy scalar equation to incorporate non-ideal gas effects and as a transported scalar to invert the temperature. Five additional species mass fraction scalars were transported in the reacting cases to accurately compute the mixture density. Scalar transport diffusivity and viscosity were calculated based on the Chung et al. method $^{17}$ from the Chapman-Enskog theory as explained in the previous section. Mixing rules from the corresponding method were also used.

\section{II..1. Chemistry model}

This study uses multi-step chemistry combustion model where kinetic mechanism, thermodynamic, and transport parameters are adapted from $\mathrm{CH}_{4}$-BFER Mechanism. ${ }^{22}$ This mechanism is validated for the conditions in this study except for pressure that is validated only until 12 atm while the operating condition for a high efficiency supercritical gas-turbine combustor is at $200 \mathrm{~atm}$. Given that no comprehensive mechanism exists for such conditions, and the observation by Franzelli et al. ${ }^{22}$ that at high starting temperatures $(\sim 700 \mathrm{~K})$, large discrepancies occur only at the low pressures, it is plausible that this model is accurate for such high pressure conditions. The mechanism consists of two global reactions below and 5 species $\left(\mathrm{CH}_{4}\right.$, $\mathrm{O}_{2}, \mathrm{H}_{2} \mathrm{O}, \mathrm{CO}, \mathrm{CO}_{2}$ ) with transport properties similar to that of GRI 3.0 mechanism.

$$
\begin{gathered}
\mathrm{CH}_{4}+1.5 \mathrm{O}_{2}=>\mathrm{CO}+2 \mathrm{H}_{2} \mathrm{O} \\
\mathrm{CO}+0.5 \mathrm{O}_{2}<=>\mathrm{CO}_{2}
\end{gathered}
$$




\section{Results}

This section is divided into two phases: 1) Numerical validation using existing experimental data to demonstrate the accuracy of the variable density solver to model supercritical regimes, 2) DNS modeling of a supercritical (i) coflowing jet, and (ii) annular jet.

\section{III.A. Coflow Jet Validation Study}

The main validation study is based on the experimental configuration of Mayer et al. ${ }^{23}$ This configuration injects supercritical nitrogen through a single, axisymmetric cylindrical injector into a chamber with quiescent, ambient temperature nitrogen gas. Table 1 shows the flow conditions used for this validation study. This experimental condition permits investigation of pressure, temperature, and inflow velocity on the characteristics of the supercritical coflowing jet.

Figure 2 shows the density contour plot with a large density change across the shear layer. Interestingly, the jet core penetrates nearly ten jet diameters before breaking down due to turbulence interactions. Note that jet penetration is highly dependent on the inflow turbulence levels. Even though the experiments do not provide detailed inflow measurements of velocity, the ability to predict jet penetration indicates that the use of a fully developed pipe flow profile at the inflow is reasonable for this configuration.

The centerline density profile (Figure 3) and its decay rate is predicted well using the modified EOS and thermodynamic parameters. Dispersion of the supercritical jet at different axial distances from the inlet is very well predicted which points to a large part, an accurate specific heat prediction close to the critical point. As temperature decreases, specific heat, $\mathrm{C}_{p}$ increases exponentially and peaks close to the critical point. ${ }^{18,24}$ The accurate location and magnitude of this $\mathrm{C}_{p}$ allows density dissipation to be predicted accurately i.e. denser flow with temperature close to the critical point will require more heat energy before density is dissipated, resulting in longer core density length and vice versa.

Table 1. Supercritical $N_{2}$ injection experimental target conditions for Case 4 in Mayer et al. ${ }^{23}$

\begin{tabular}{rrrrr}
$\mathrm{P}(\mathrm{MPa})$ & $U_{\text {jet,builk }}(\mathrm{m} / \mathrm{s})$ & $T_{\text {jet }}(\mathrm{K})$ & $T_{c o}(\mathrm{~K})$ & $\mathrm{D}(\mathrm{mm})$ \\
\hline 4.0 & 5.0 & 130.0 & 298 & 2.2
\end{tabular}

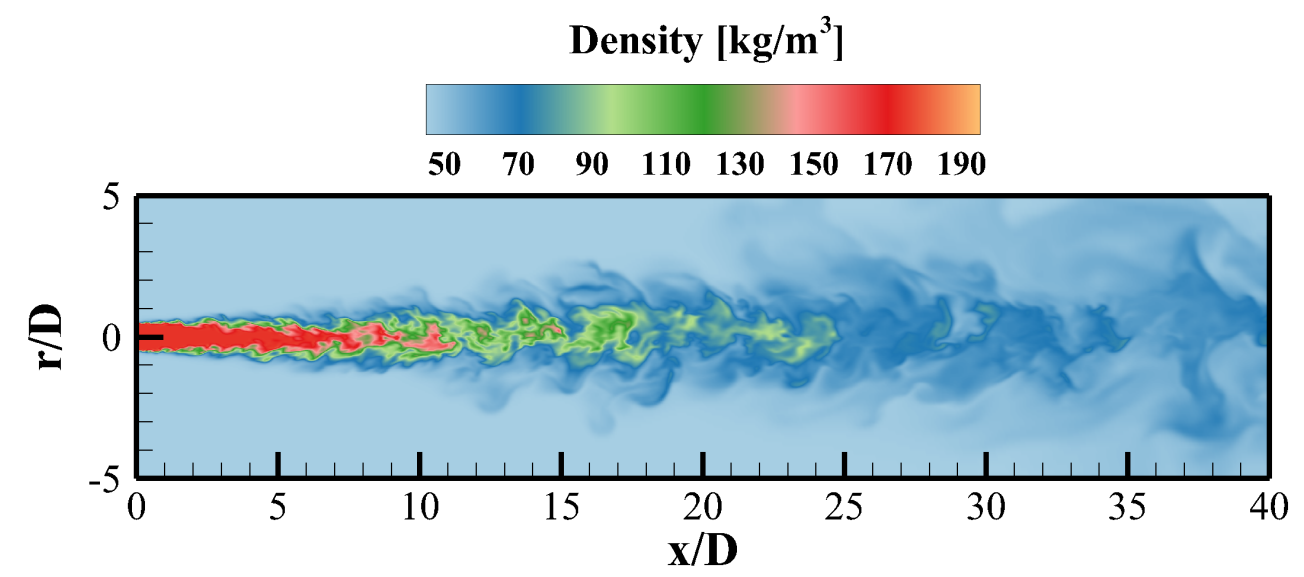

Figure 2. $\mathrm{N}_{2}$ Density; Target properties: $4 \mathrm{MPa}, 5 \mathrm{~m} / \mathrm{s}, 130 \mathrm{~K}$

\section{III.B. Coflow Reacting Jet}

DNS study of a coflow reacting jet pertinent to realistic gas-turbine conditions is studied here. The flow field is characterized by a methane jet at ambient condition being injected into a coflowing supercritical mixture 


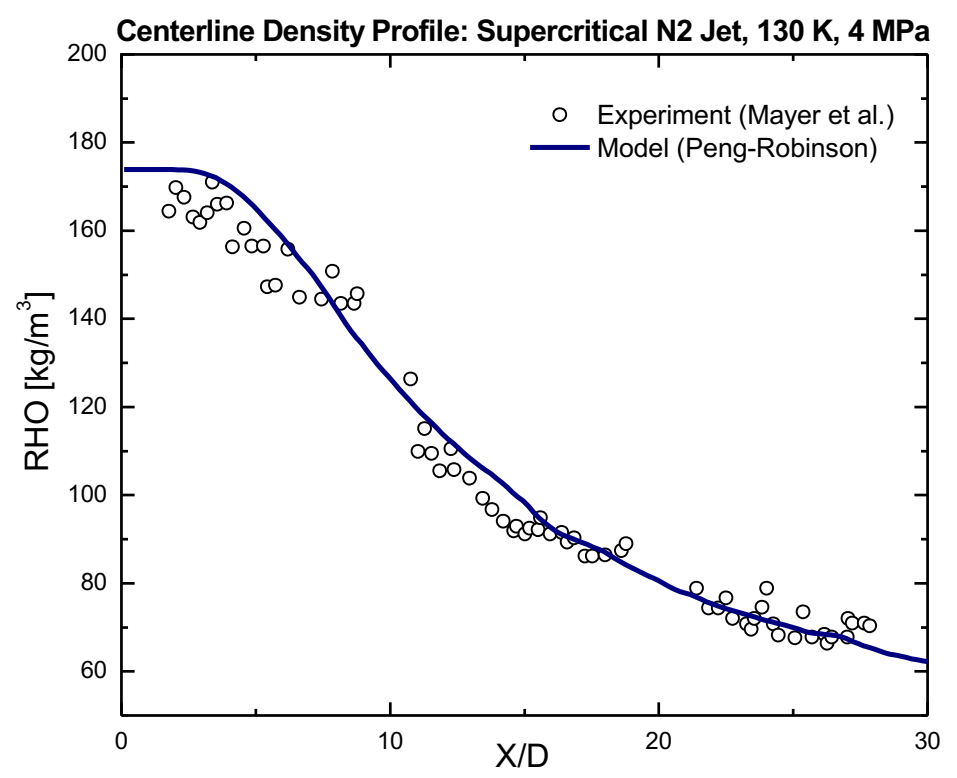

Figure 3. Centerline $\mathrm{N}_{2}$ Density: $4 \mathrm{MPa}, 5 \mathrm{~m} / \mathrm{s}, 130 \mathrm{~K}$

of $\mathrm{CO}_{2} / \mathrm{O}_{2}$. Details of the flow conditions are listed in Table 2. The Re w.r.t. the fuel jet diameter is 4,000 with the smallest mesh size at $2.0 \times 10^{-5} \mathrm{~m}$ to resolve the Kolmogorov length scale. This smallest Kolmogorov length scale, $\eta$, is calculated using experimental data of kinetic energy dissipation, $\epsilon$, from Panchapakesan and Lumley $(1993)^{25}$ and $\eta$ is related to the axial length as shown in Boersma et al. ${ }^{26}$ This way, $\eta$ is directly related to the downstream location by jet diameter, $D$. For this DNS, $\eta$ is $0.04 \mathrm{D}$ which for $20 \mathrm{D}$ location and downstream, all gridspacings are of the same order of $\eta$, therefore resolving sufficient energy of the energy spectrum. The domain contains 20 million grid points with axial and diameter size selected to be $80 \mathrm{D} \times 20 \mathrm{D}$ with $\mathrm{D}=$ diameter of jet inlet. Such operating conditions are chosen based on conditions that are commonly encountered in a supercritical gas-turbine combustor with $\phi=1.1$ and $T_{\text {burnt }}=T_{\text {turbine }} \sim 1600 K^{6}$ where $T_{\text {burnt }}$ is the burnt gas temperature in combustor while $T_{\text {turbine }}$ is the turbine inlet temperature.

The chemical reaction is solved using a 5-species 2-step mechanism as explained in section II..1. Note that the initial conditions here are in the supercritical region, not close to the critical point for any of the species considered. However, the application studied here, a gas-turbine combustor, require transcritical regime to be available as well for the mixture and for parameters used in calculating departure from ideal gas thermodynamic properties as explained in section I.A.2.

From the instantaneous plots of temperature (Fig.4) and diffusivity (Fig.5), we can see that the jet flow is being enveloped by a thin flame-front that is attached close to the lip of the jet. Furthermore, as a result of the $\mathrm{CO}_{2}$ dilution, the temperature peaks at a fairly low value $T_{\max } \sim 1490 \mathrm{~K}$. The high $C_{p}$ value of the $\mathrm{sCO}_{2}$ diluent and well premixed coflow with $\mathrm{O}_{2}$ results in low temperature at the flame front.

DNS data is then studied by comparing to 1-D laminar counterflow diffusion flame solutions obtained from FlameMaster. ${ }^{27}$ The counterflow diffusion flame solution in mixture fraction space is a well-known topic, ${ }^{28}$ and details for solving the counterflow diffusion flame is omitted here for brevity. The 1-D diffusion flame calculation is done without using supercritical models for thermodynamic properties. This is to compare the calculations from the original FlameMaster modules to DNS data and to determine if the original flamelet method is a good representation of supercritical combustion.

A general layout showing temperature in mixture fraction space is shown in l.h.s of Fig.6, where a scatter plot DNS data is overlayed with flamelet solutions. For visualization purposes, on the l.h.s. of Fig.6, the DNS scatters are only plotted 1 for each 100 cell index, and on the r.h.s. of Fig.6, only two flamelet solutions with the chosen $\chi_{s t}$ values are plotted. The definition of $Z_{m i x}$ here is the Bilger mixture fraction. ${ }^{29}$ It can be seen from Fig.6 that not only does most of the DNS data fall into the regime covered by the flamelet solutions, but also the DNS scatter has shown a certain preference of concentration in $T-Z_{m i x}$ space. Further, from 
the conditioned average plot on the r.h.s of Fig.6, in the region close to the jet (small mixture fraction $Z_{\text {mix }}$ ), it can be seen that the flamelet results with the corresponding $\chi_{s t}$ achieved flame temperatures reasonably close to the average temperature encountered in DNS. However for the fuel side, there can be found an over-prediction of flamelet temperature comparing to the DNS average conditioned on the corresponding $\chi_{s t}$ value. The conditioned averages are calculated from all the computational cells, and since $\chi$ is locally accessible variable, the corresponding $\chi_{s t}$ value are back calculated using the standard profile between $\chi, \chi_{s t}$ and $Z_{m i x}$, which will be discussed more later in this section. The discrepancy between DNS and flamelet temperature may indicate one of the two possibilities. Firstly, the DNS scatter at large mixture fraction $Z_{m i x}$ still falls into the regime covered by the flamelet solution, noticing that after a certain extent, the lower flamelet curves should correspond to the intermediate and extinction branch if plotted on diagram showing the S-shaped curve. This may indicate that the supercritical flame is more sensitive to strain rate on the fuel side, so that the DNS scatters with scalar dissipation rate $\chi_{s t}$ lower than the flamelet extinction scalar dissipation rate are already operating under the transition and extinction mode. Secondly, since the standard profile (see Eq.28) is applied to obtain $\chi_{s t}$ based on $\chi$, it may also be possible that it is the over-prediction of $\chi$ on the fuel rich side in the standard profile of $\chi$ that results in an insufficient averaging of the DNS flame temperature conditioned on $\chi$. And as can be seen in remaining paragraphs, the latter is also supported by the $\chi-Z_{m i x}$ relation plot of the DNS data (Fig.9), which is considered here as a more preferred aspect to explain such relation between the DNS and the flamelet results.

Table 2. Operating conditions for reacting jet with annulus case

\begin{tabular}{rrrrrr} 
Inlet & $U(\mathrm{~m} / \mathrm{s})$ & $T(\mathrm{~K})$ & $D(\mathrm{~mm})$ & $P(\mathrm{MPa})$ & Species Mass frac. $\left(Y_{i}\right)$ \\
\hline Jet & 1.0 & 300 & 0.50 & 20 & $\mathrm{CH}_{4}(1.0)$ \\
Coflow & 0.1854 & 1100 & 10.0 & 20 & $\mathrm{O}_{2}(0.0457)+\mathrm{CO}_{2}(0.9543)$
\end{tabular}

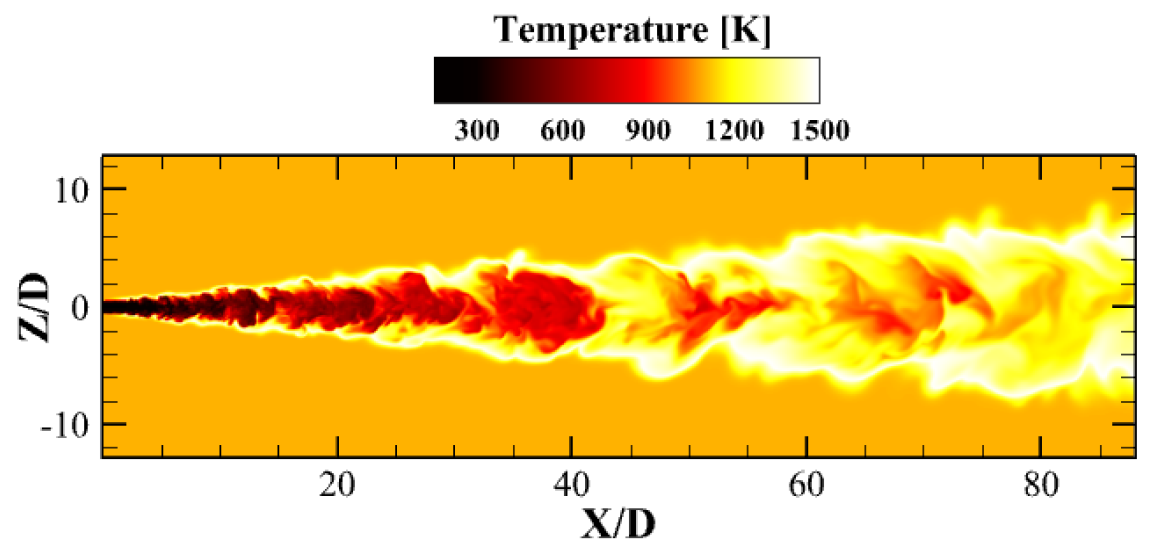

Figure 4. Temperature instantaneous contour plot of reacting jet with $\mathrm{CO}_{2}$ diluent

The supercritical coflow jet flame is further studied in terms of the species mass fractions, as shown in Fig.7, where $Y_{C O}$ and $Y_{H 2 O}$ are plotted against $Z_{m i x}$ colored by their chemical source term value. Previously, it was mentioned that on the fuel side, the flame temperature was over-predicted in the 1D-flamelets solutions compared to the conditional temperature average from the DNS. Here in Fig.7, such over-prediction is again found for the CO mass fraction. This is also confirmed by the source term values indicated by the color, and notice that the range of colormap for the scatter is much smaller than that for the flamelet solutions in Fig.7. This indicates that most the CO formation takes place under very low strain rate and is rarely seen in the DNS scatter on the oxidizer side and almost never seen on the fuel side. Combustion on the fuel side is more clearly revealed by the $\mathrm{H}_{2} \mathrm{O}$ mass fraction plot on r.h.s. of Fig.7, where it can be seen the scatters are mostly concentrated below the cluster of flamelet solutions that represent the upper branch. Although it is not shown here, but it is known that the sparsely located flamelet curves corresponds to those on the intermediate branch, therefore the $\mathrm{H}_{2} \mathrm{O}$ mass fraction scatter indicate that a considerable amount of the DNS flame is actually operating in the transition region. One of the reason behind this is due to the dilution of large amount of $\mathrm{CO}_{2}$, which reduces the reaction rate, resulting in a combustion process that is 


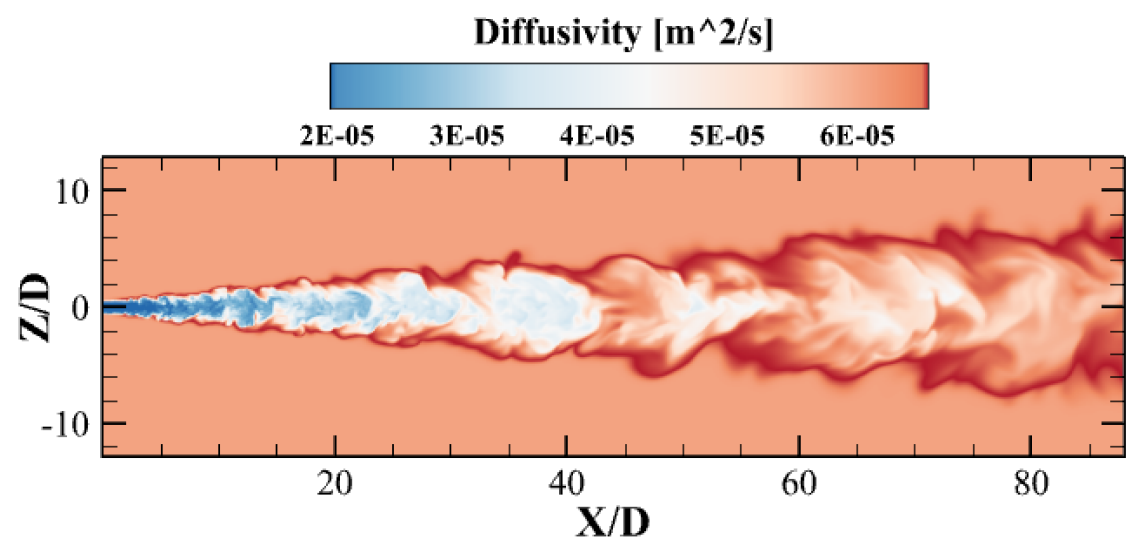

Figure 5. Diffusivity instantaneous contour plot of reacting jet with $\mathrm{CO}_{2}$ diluent
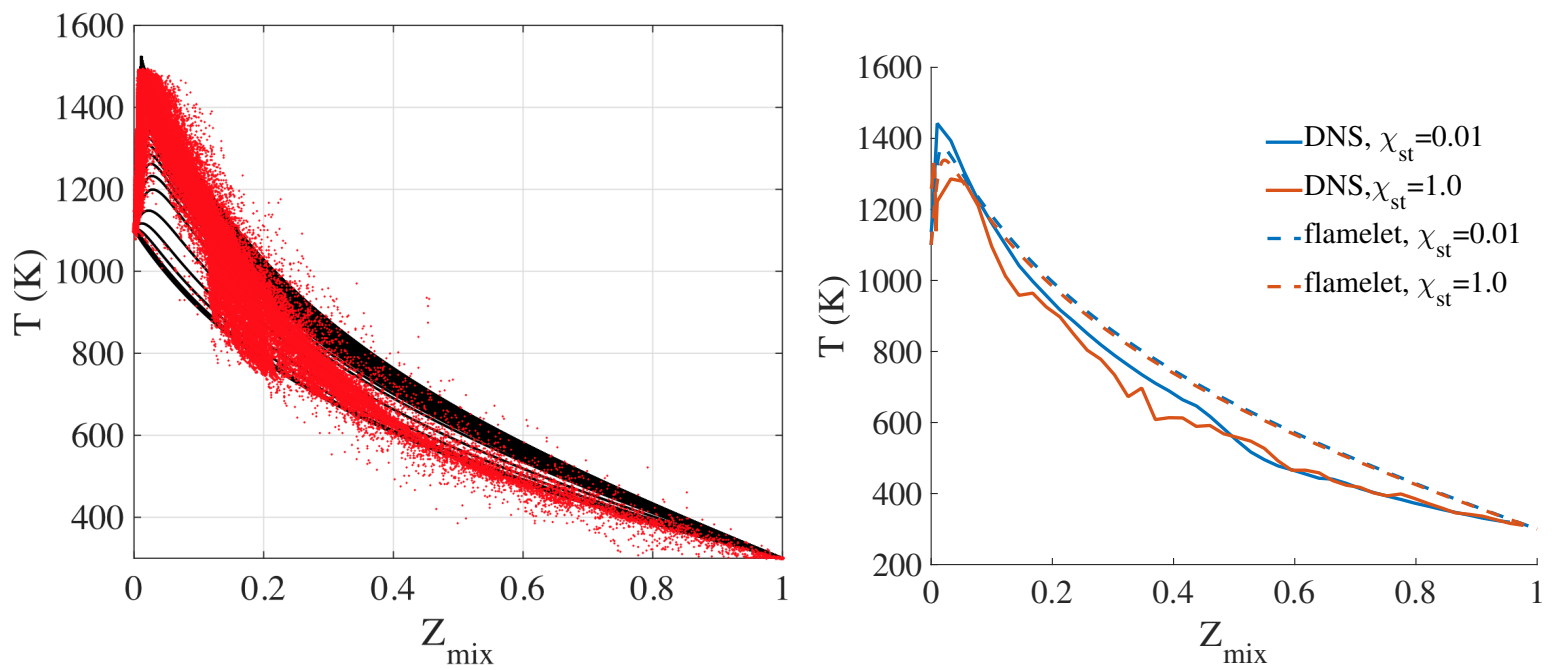

Figure 6. Temperature- $Z_{m i x}$ data in DNS overlayed with flamelet calculations. On the l.h.s., the DNS data is plotted in terms of instantaneous scatter, and the r.h.s. in terms of conditional averaged value based on $\chi_{s t}$

very sensitive to the flow mixing and strain rate.

In Fig.8, heat production rate and $\mathrm{CO}_{2}$ mass fraction are plotted against $Z_{m i x}$. The peak of heat release is only achieved under very low scalar dissipation rate and is rarely encountered in the DNS data. The reason for DNS data not achieving the same peak value for heat release as flamelet may be due to strain effects. However, since the peak region is very narrowed down to near $Z_{m i x, s t}$, the peak temperature from the DNS data still ends up being very close to the unstrained flamelet calculation. $\mathrm{CO}_{2}$ is mainly acting as a diluent in the coflow, and therefore its distribution is mostly linear with relation to the mixture fraction, except for $Z_{m i x} \sim Z_{m i x, s t}$.

Another interesting finding for the coflow jet is that when plotting the DNS averaged scalar dissipation rate in mixture fraction space, the peak is achieved near the value of $Z_{m i x}=0.4$ in the upstream near the jet exit but as the reacting flow moves downstream, comparison between location of the peaks between the flamelet counterflow diffusion flame calculations and the DNS data shows increasing agreement. Note that after $\mathrm{x} / \mathrm{D}=15$ downstream location, there are no higher mixture fraction than $Z_{m i x}=0.4$, which is because at downstream fuel rich pockets from the jet stream are diluted through the mixing process. In Fig.9, the $\chi$ for both DNS results and a standard solution for $\chi-Z_{\text {mix }}$ relation with $\chi_{s t}=0.45$ are plotted in comparison, where it can be seen that the slope and peak values are similar for the fuel lean side, actually the peak value from the DNS data is shifted slightly toward fuel lean side. As a brief explanation, the standard $\chi-Z_{m i x}$ relation is obtained from a strained 1-D diffusion flame by solving the flamelet equations in 1-D similarity coordinate space assuming infinite fast chemistry and constant density for the momentum equation, ${ }^{28}$ and 

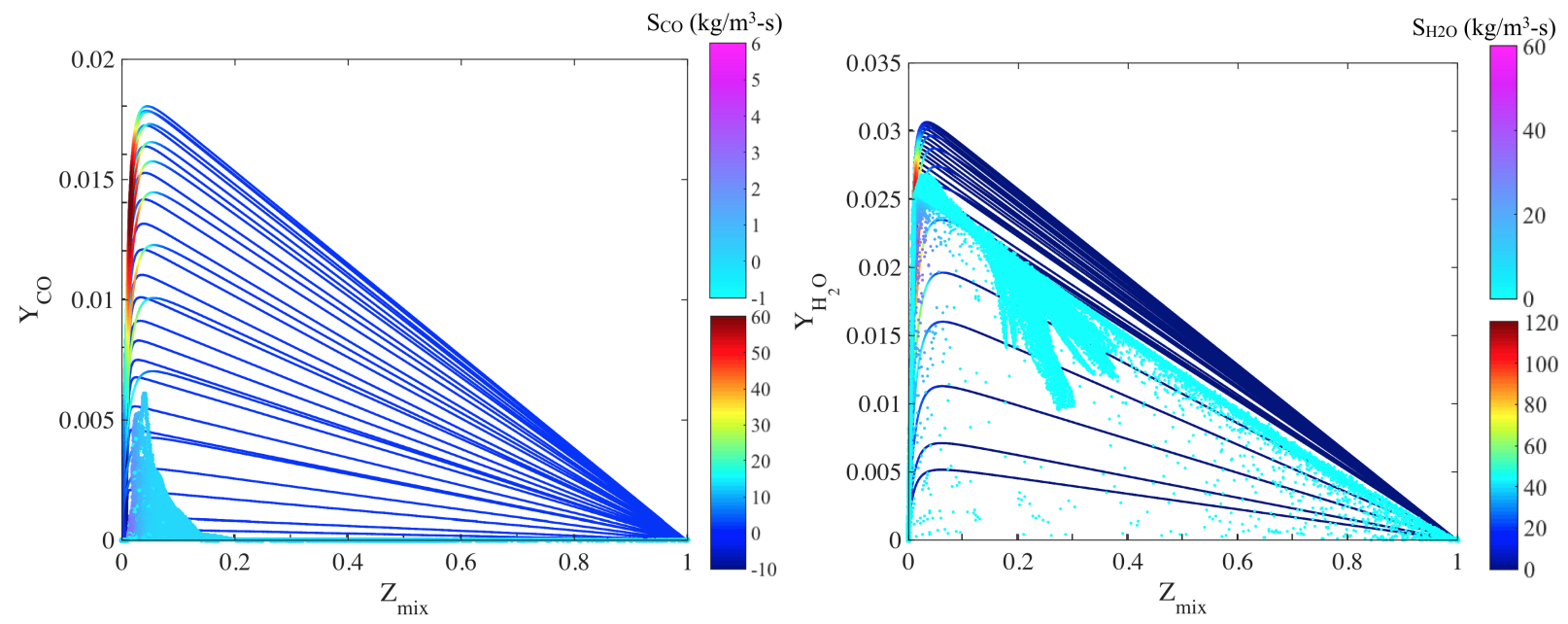

Figure 7. Species mass fraction- $Z_{m i x}$ DNS data scatter plot overlayed with flamelet calculations, both DNS scatter and flamelet curves are colored by the species' chemical source for mixture fraction $Z_{m i x}$, while using different colormaps.

the solution profile is the well-known error function relation between $\chi$ and $Z_{m i x}$ in Eq.28, which is also the profile applied when solving the counterflow diffusion flamelets in the mixture fraction space.

$$
\chi=\frac{a_{s}}{\pi} \exp \left(-2\left[\operatorname{erf}^{-1}\left(1-2 Z_{m i x}\right)^{2}\right]\right),
$$

where $a_{s}$ is the velocity gradient at stagnation point, and in this case chosen to be $a_{s}=355 \mathrm{~s}^{-1}$ to match the DNS peak sampling at the plane $x / D=3$. The assumption of constant density for the flamelet momentum equation could be the reason why the DNS data peak is shifted slightly toward the fuel lean side, and is further modified by Kim and Williams ${ }^{30}$ in Eq.29

$$
\chi=\frac{a_{s}}{4 \pi} \frac{3\left(\sqrt{\frac{\rho_{\infty}}{\rho}}+1\right)^{2}}{2 \sqrt{\frac{\rho_{\infty}}{\rho}}+1} \exp \left(-2\left[\operatorname{erf}^{-1}\left(1-2 Z_{\text {mix }}\right)^{2}\right]\right),
$$

where the density ratio is obtained from the flamelet solution solved in mixture fraction space using detailed chemistry. And it turns out the modified peak with Eq.29 is only slightly shifted to the left of $Z_{\text {mix }}=0.5$. Notice that other assumptions such as $\rho^{2} D=$ const (Chapman gas approximation) are still applied to obtain Eq.29. Although those assumptions are usually valid for counterflow diffusion flame, at this point they becomes one of the possible cause for the shift of the DNS peak toward $Z_{\text {mix }}=0$ from both of the standard profiles, and remain to be investigated for supercritical combustion.
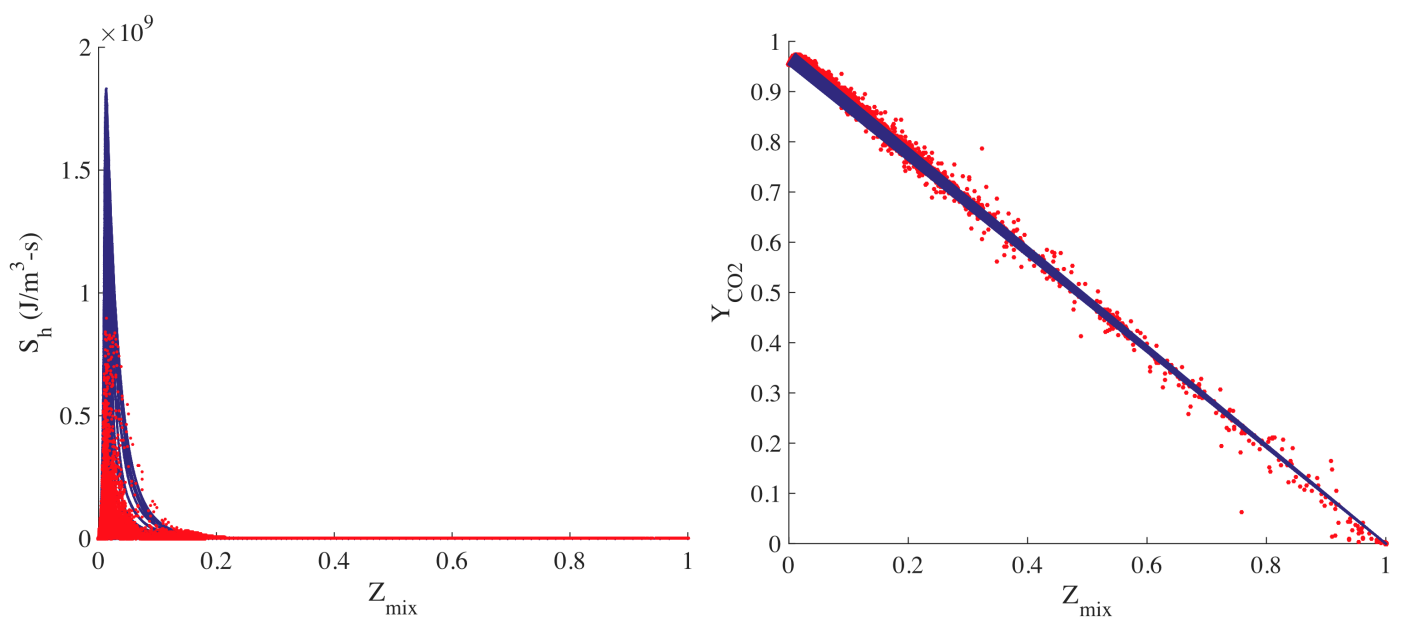

Figure 8. Enthalpy source (left) and $Y_{\mathrm{CO}_{2}}$ (right) - $Z_{m i x}$ DNS data scatter plot overlayed with flamelet calculations. 


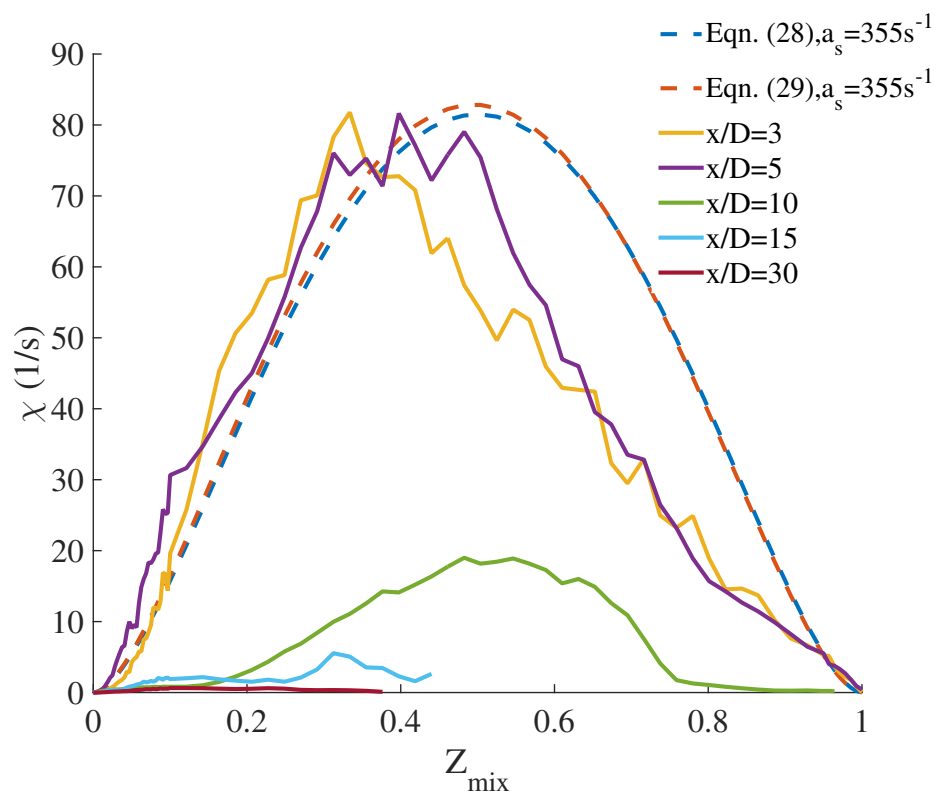

Figure 9. Scalar dissipation rate vs. $Z_{m i x}$. Solid line represents a steady strained 1-D diffusion flame with infinitely fast chemistry, dashed-line is obtained by averaging DNS data based on $Z_{m i x}$ at different downstream locations

A cause for concern to the operation of a turbine is the concentration of $\mathrm{CO}$ species entering the turbine from the combustor. Even a small mass fraction of $\mathrm{CO}$ can potentially react with coolant air at the tip of the turbine blade to produce $\mathrm{CO}_{2}$ which will generate a large amount of heat release and thus reduce the material life-expectancy in the long-run. In this DNS case, the mass fraction of CO species is looked into and the instantaneous as well as time-averaged plots are shown in Fig.10. As shown, the mass fraction of CO is mostly concentrated at the flame front and at pockets of fuel rich region as plotted in Fig.6. Time-averaged data also shows a moderately high concentration of $\mathrm{CO}$ at the downstream region $60<X / D<88$. Future design of supercritical direct-fired combustor will have to look into this factor when considering methods for decreasing $\mathrm{CO}$ mass fraction from the combustion process. Future work will also concentrate on testing the effect of different scalar dissipation rates and fuel injection methods to decrease the probability of fuel rich regions of high temperature igniting and producing $\mathrm{CO}$.

\section{III.C. Annular Reacting Jet}

Another configuration that is important to realistic gas-turbine design conditions is a jet-in-crossflow configuration. Oxygen and methane streams enter through separate nozzles while supercritical- $\mathrm{CO}_{2}$ serves as the crossflow. In order to replicate the flame physics in this geometry, a simpler annular jet is considered here. Here, a diluted $\mathrm{O}_{2}+\mathrm{CO}_{2}$ stream flows in parallel to the fuel jet, with a coflow of supercritical $\mathrm{CO}_{2}$ enveloping the domain. Same general configuration and global stoichiometry are chosen for this case, compared to the coflowing jet case, corresponding to $R e=4,000$ with 20 million grid points and similar mesh configuration as shown in Table 3.

Table 3. Operating conditions for reacting jet with annulus case

\begin{tabular}{rrrrrr} 
Inlet & $U(\mathrm{~m} / \mathrm{s})$ & $T(\mathrm{~K})$ & $D(\mathrm{~mm})$ & $P(\mathrm{MPa})$ & Species Mass frac $\left(Y_{i}\right)$ \\
\hline Jet & 1.0 & 300 & 0.50 & 20 & $\mathrm{CH}_{4}(1.0)$ \\
Annulus & 5.061 & 1100 & 1.50 & 20 & $\mathrm{O}_{2}(0.1538)+\mathrm{CO}_{2}(0.8462)$ \\
Coflow & 0.1854 & 1100 & 10.0 & 20 & $\mathrm{CO}_{2}(1.0)$
\end{tabular}

The aim of this case is to understand the combustion process of a different configuration to the coflowing jet. As expected of an annular case with high annular velocity compared to the jet velocity, the combustion is highly mixing-controlled and depends on the flow turbulence at the downstream location from the inlet. From Fig.11 below, fuel $/ \mathrm{O}_{2} /$ supercritical $-\mathrm{CO}_{2}$ mixture is shown to ignite at $X / D=20$ location downstream 

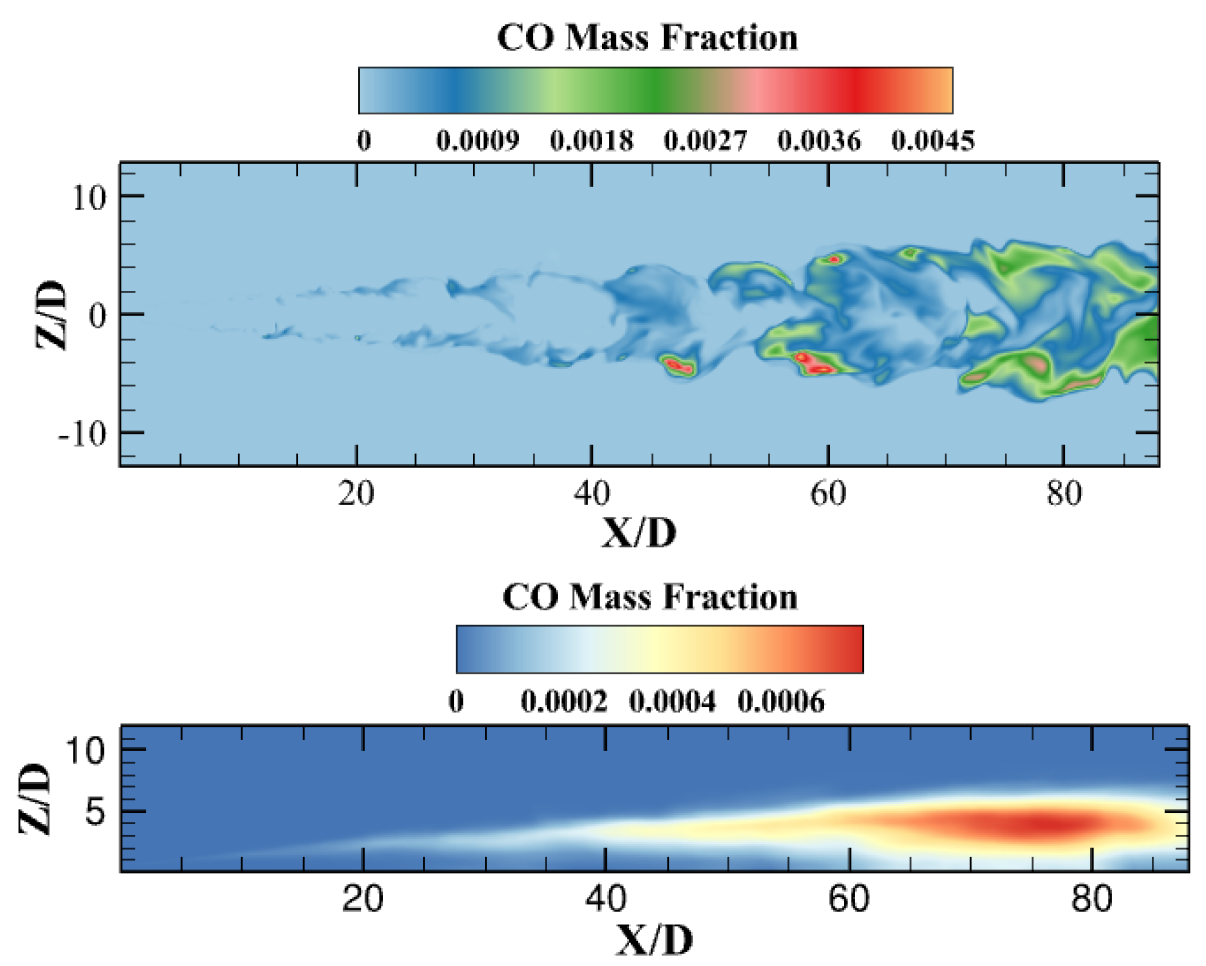

Figure 10. CO mass fraction instantaneous and time-averaged plot of reacting jet with $\mathrm{CO}_{2}$ diluent

of the inlet, where the flame is lifted and not attached to the lip in the jet coflow case. This will prevent any damage to the inlet wall but causes difficulties in controlling the flame because of the strong diffusion and strain-rate based flame ignition. Another disadvantage of the annular case is the high maximum temperature $(\sim 1900 K)$ of the flame compared to the jet case $(\sim 1500 K)$ which could propagate and result in high local temperature that could damage the turbine blades and result in lowered turbine efficiency.

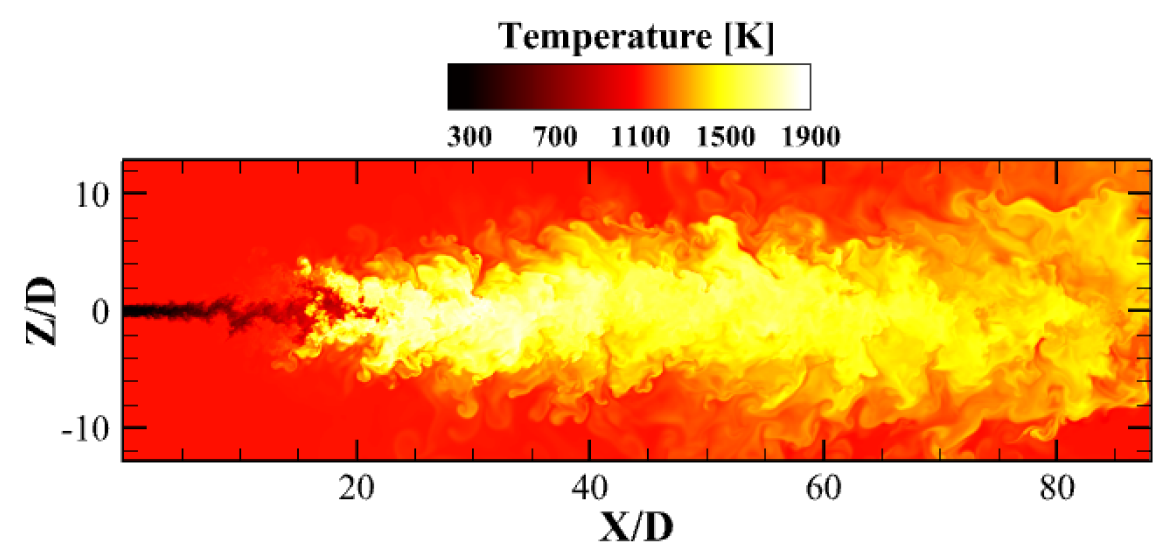

Figure 11. Temperature instantaneous contour plot of reacting jet with annular and $\mathrm{CO}_{2}$ diluent

As there are three different streams in the system of the annular jet case, an additional mixture fraction is introduced, and distinguished using subscript number. Here $Z_{1}$ indicates the proportion of mass flow originating from the jet stream, and $Z_{2}$ is assigned for the annular jet. Rigorously, there is also a $Z_{3}$ for the coflow, however by definition it automatically follows the relation $Z_{3}=1-\left(Z_{1}+Z_{2}\right)$, and the domain of mixture fraction space on the $Z_{1}-Z_{2}$ plane should be a unit triangle. An example of the transformation into the two mixture fraction space can be seen in Fig.12, where the DNS data of the original computational domain is plotted in scatter and colored by an extra property in mixture fraction space. 

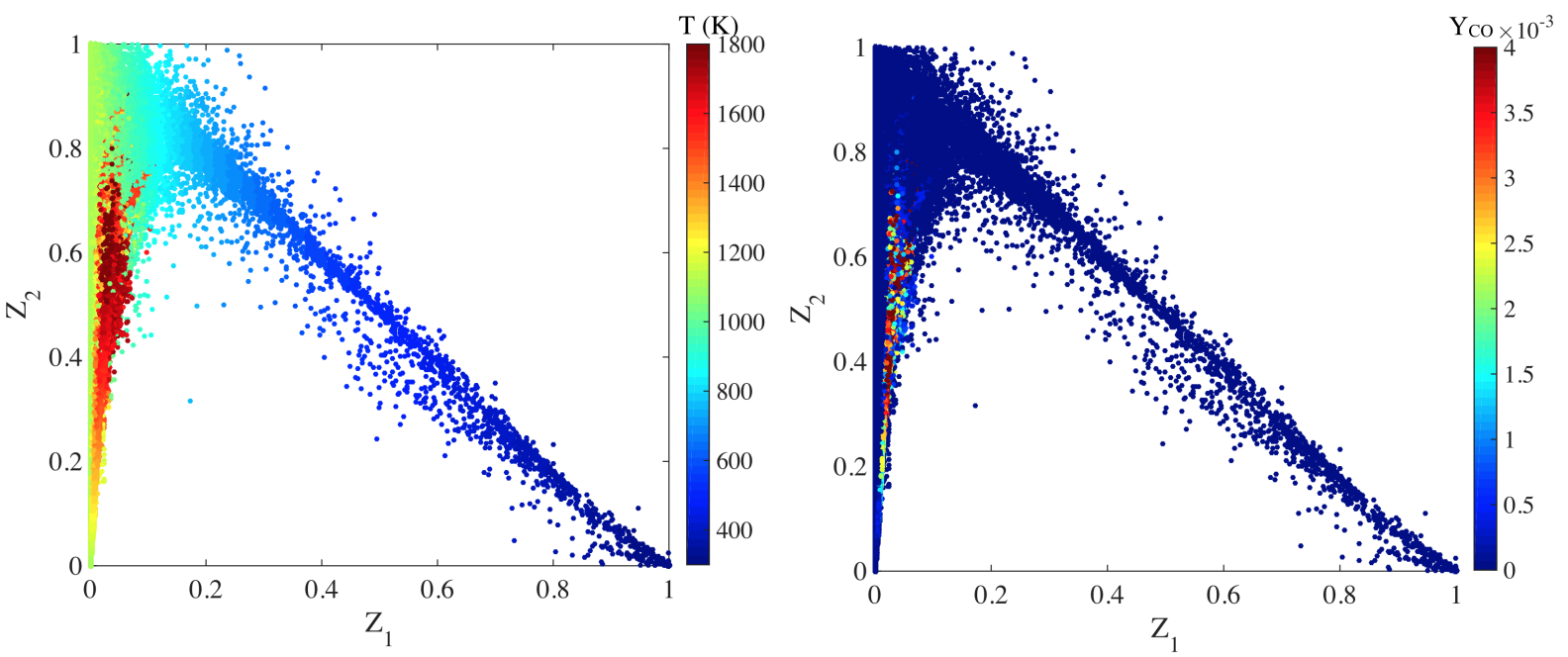

Figure 12. Instantaneous scatter plot of DNS computational domain in $Z_{1}-Z_{2}$ space, colored by temperature (left) and CO mass fraction (right)

In Fig.12 it can be seen that the direction of the most obvious temperature variation is in $Z_{1}$, which is because $Z_{1}$ indicates the mixing between the fuel jet and the rest of the mixture, plus that the jet stream has the lowest temperature of $300 \mathrm{~K}$ compared to the rest of the inlets. A high concentration of high temperature regions is achieved in the region of of $Z_{1} \sim 0.05$ and $Z_{2} \sim 0.6$, and the peak value of temperature $1800 \mathrm{~K}$ is higher than the previous coflow case of $1500 \mathrm{~K}$, which is because the fuel stream is now able to access a higher concentration of oxidizer from the annular jet. The influence of $Z_{2}$ comes into place mostly when $Z_{1}$ decreases to around 0.1 , by changing the proportion of mixture provided for the reaction. For $Z_{1}>0.2$ and $Z_{2}>0.8$, the variation of temperature is mainly driven by mixing. Another distinguishable observation from Fig.12 is the triangle-shaped region that is rarely encountered by the DNS data, which shows a pattern of how $Z_{1}$ and $Z_{2}$ is linearly correlated in the DNS case. As an explanation, such pattern should be governed by the inner and outer mixing layer of the annular jet, where the inner mixing layer should correspond to the left "edge" of the "triangle", and the outer mixing layer the right "edge".

Comparing $\mathrm{CO}$ species mass fractions with the jet case, the magnitude of $\mathrm{CO}$ mass fraction produced is an order of magnitude higher than the jet case. Furthermore, the location of peak $\mathrm{CO}$ mass fraction for both cases are also different with the annular case experiencing a higher $\mathrm{CO}$ mass fraction close to the axis at $X / D=40$. This is predominantly due to the inhomogeneous mixing between the fuel and oxidizer resulting in local regions corresponding to $Z_{1}<0.05$ and $Z_{2} \sim 0.6$ on the r.h.s. plot in Fig.12. This region where there is less dilution from $\mathrm{CO}_{2}$ has a high temperature due to the lowered mixture $C_{p}$ values. The local fuel rich condition in the central axis is due to the enveloping effect of the annular $\mathrm{O}_{2}$ jets preventing the central fuel jet from diffusing radially downstream. This high strain rate region produces incomplete combustion between the fuel and oxidizer, which produces a high concentration of $\mathrm{CO}$ species.

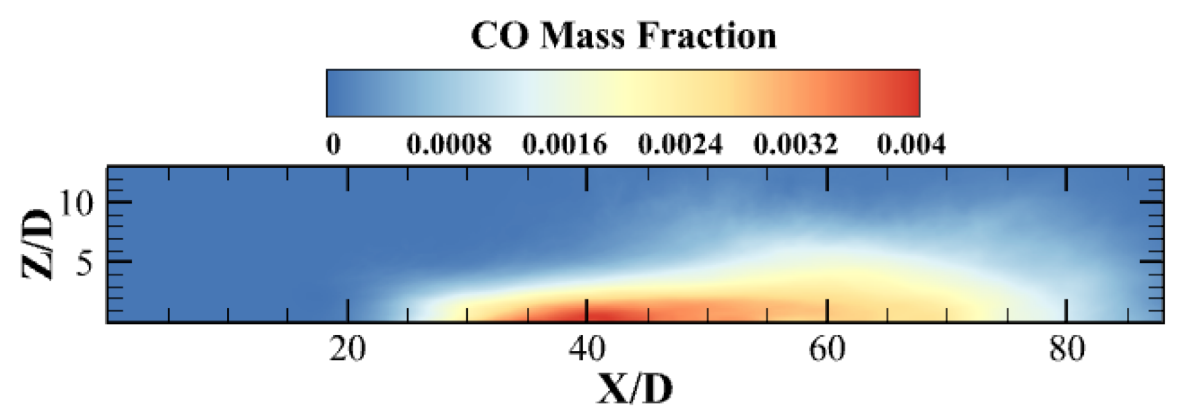

Figure 13. CO species time-averaged mass fraction contour plot of reacting jet with annular and $\mathrm{CO}_{2}$ diluent 


\section{Conclusion and Future Work}

In this study, two possible configurations of supercritical combustion in a gas-turbine combustor are being simulated and analyzed - (1) jet with coflow, and (2) jet with annular. We also implemented real gas cubic equations and departure functions for thermodynamic and transport properties to a low-Mach solver. Detailed representation of the algorithm and Newton iteration to arrive at the final mixture temperature and density from transported enthalpy and species mass fractions is in Fig. 1. For reacting DNS simulations, a two-step chemistry approach is being implemented for $\mathrm{CH}_{4}+\mathrm{O}_{2}$ combustion with $\mathrm{CO}_{2}$ as diluent. A lower peak temperature is being predicted due to the high concentration of dilution from $\mathrm{CO}_{2}$, a high $C_{p}$ species. This work also illustrates the capability of this in-house code to investigate a non-reacting validation case by Mayer et al. ${ }^{23}$ where $\mathrm{N}_{2}$ mixing in a quiescent chamber close to the critical point compares favorably with the experimental results. The simulation result shows accurate diffusivity calculations from comparisons to the centerline density.

From analysis of the DNS data for both reacting cases, a clear distinction between the configuration at supercritical conditions is seen. A simple fuel jet with premixed (oxidizer+diluent) coflow case shows an attached flame at the lip of the fuel jet inlet with low maximum flame temperature mainly due to the high rate of dilution by $\mathrm{CO}_{2}$. This condition is advantageous to the operating condition of a direct-fired supercritical combustor where fuel and oxidizer is being injected into a supercritical flow to heat up the working fluid because the maximum possible temperature for optimum efficiency of the turbine expansion phase is $\sim 1500 K$. Furthermore, formation of $\mathrm{CO}$ in the jet configuration is at the flame front further downstream of the jet inlet and shows a low amount of $\mathrm{CO}$ being produced, indicating a close to complete combustion process in this configuration.

For the jet with annular case, the approximately $85 \% \mathrm{CO}_{2}$ and $15 \% \mathrm{O}_{2}$ (by mass) annular jet produces a highly turbulent region downstream at $X / D=20$, producing a highly lifted flame with high flame temperatures approaching 1900K. Moreover, an order of magnitude higher CO mass fractions is also observed in the annular case compared to the jet case mainly due to the high temperature at the central axis region increasing the reaction rate of $\mathrm{CO}$ formation. Fuel rich conditions at the central mixing zone due to inhomogeneous mixing also decreases the rate of $\mathrm{CO}$ being turned into $\mathrm{CO}_{2}$, thus forming an accumulation of $\mathrm{CO}$ species at the central region. This combination of higher maximum temperature and higher $\mathrm{CO}$ mass fraction will inevitably lead to unfavorable conditions for the operation of the turbine where CO species will react with dilution air at the tip of the turbine blade to complete the combustion process and produce large amounts of heat release that will decrease the life-cycle expectancy of the turbine blades.

In the future, the authors plan to conduct more studies into a different configuration that is still pertinent to normal combustor operations to determine if these conditions are conducive to a supercritical environment. An example of such configuration is the Jet-In-Crossflow configuration where high strain rate crossjet mixing is expected to enhance the combustion process that could further reduce $\mathrm{CO}$ species production perhaps to a level even lower than that of the jet case studied here. Furthermore, the DNS data obtained here can be a good yardstick for future development of algorithms and combustion models for the supercritical regime. This work also pushes for more simulation work on transcritical combustion where large density gradient with small temperature gradient is especially important in the mixing region prior to combustion. 


\section{References}

${ }^{1}$ Yang, V., "Modeling of supercritical vaporization, mixing, and combustion processes in liquid-fueled propulsion systems," Proceedings of the Combustion Institute, Vol. 28, No. 1, 2000, pp. 925-941.

${ }^{2}$ Oefelein, J. C. and Yang, V., "Modeling High-Pressure Mixing and Combustion Processes in Liquid Rocket Engines," Journal of Propulsion and Power, Vol. 14, No. 5, 1998, pp. 843-857.

${ }^{3}$ Masi, E., Bellan, J., , K. G., and Okong'o, N. a., "Multi-species turbulent mixing under supercritical-pressure conditions: modelling, direct numerical simulation and analysis revealing species spinodal decomposition," Journal of Fluid Mechanics, Vol. 721, 2013, pp. 578-626.

${ }^{4}$ Borghesi, G. and Bellan, J., "Irreversible entropy production rate in high-pressure turbulent reactive flows," Proceedings of the Combustion Institute, Vol. 35, No. 2, 2015, pp. 1537-1547.

${ }^{5}$ Lacaze, G. and Oefelein, J. C., "A non-premixed combustion model based on flame structure analysis at supercritical pressures," Combustion and Flame, Vol. 159, No. 6, 2012, pp. 2087-2103.

${ }^{6}$ McClung, A., Brun, K., and Chordia, L., "Technical and economic evaluation of supercritical oxy-combustion for power generation," The 4th International Symposium - Supercritical CO2 Power Cycles, , No. 1, 2014, pp. 1-5.

${ }^{7} \mathrm{Kim}$, J. and Moin, P., "Application of a fractional-step method to incompressible Navier-Stokes equations," Journal of Computational Physics, Vol. 59, No. 2, 1985, pp. 308-323.

${ }^{8}$ Desjardins, O., Blanquart, G., Balarac, G., and Pitsch, H., "High order conservative finite difference scheme for variable density low Mach number turbulent flows," Journal of Computational Physics, Vol. 227, 2008, pp. 7125-7159.

${ }^{9}$ Lietz, C., Hassanaly, M., Raman, V., Kolla, H., Chen, J., and Gruber, A., "LES of Premixed Flame Flashback in a Turbulent Channel," 52nd Aerospace Sciences Meeting, , No. January, 2014, pp. 1-11.

${ }^{10}$ Moureau, V., Minot, P., Pitsch, H., and Berat, C., "A ghost-fluid method for large-eddy simulations of premixed combustion in complex geometries," Journal of Computational Physics, Vol. 221, No. 2, 2007, pp. 600-614.

${ }^{11}$ Kempf, A., Lindstedt, R. P., and Janicka, J., "Large-eddy simulation of a bluff-body stabilized nonpremixed flame," Combustion and Flame, Vol. 144, No. 1-2, 2006, pp. 170-189.

${ }^{12}$ Mueller, M. E. and Pitsch, H., "LES model for sooting turbulent nonpremixed flames," Combustion and Flame, Vol. 159, No. 6, 2012, pp. 2166-2180.

${ }^{13}$ Koo, H., Hassanaly, M., Raman, V., Mueller, M. E., and Geigle, K. P., "Large Eddy Simulation of Soot Formation in a Model Gas Turbine Combustor," Journal of Engineering for Gas Turbines and Power, Vol. 139, No. March, 2016, pp. 1-9.

${ }^{14} \mathrm{Han}, \mathrm{W}$., Raman, V., and Chen, Z., "LES/PDF modeling of autoignition in a lifted turbulent flame: Analysis of flame sensitivity to differential diffusion and scalar mixing time-scale," Combustion and Flame, Vol. 171, 2016, pp. 69-86.

${ }^{15}$ Jenny, P., Pope, S., Muradoglu, M., and Caughey, D., "A Hybrid Algorithm for the Joint PDF Equation of Turbulent Reactive Flows," Journal of Computational Physics, Vol. 166, No. 2, 2001, pp. 218-252.

${ }^{16}$ Herrmann, M., Blanquart, G., and Raman, V., "Flux Corrected Finite Volume Scheme for Preserving Scalar Boundedness in Reacting Large-Eddy Simulations," AIAA Journal, Vol. 44, No. 12, 2006, pp. 2879-2886.

${ }^{17}$ Chung, T. H., Ajlan, M., Lee, L. L., and Starling, K. E., "Generalized multiparameter correlation for nonpolar and polar fluid transport properties," Industrial \& Engineering Chemistry Research, Vol. 27, 1988, pp. 671-679.

${ }^{18}$ Congiunti, a., Bruno, C., and Giacomazzi, E., "Supercritical Combustion Properties," AIAA Aerospace Sciences Meeting and Exhibit, Vol. 41, No. January, 2003, pp. 1-20.

${ }^{19}$ Harstad, K. G., Miller, R. S., and Bellan, J., "Efficient high-pressure state equations," AIChE Journal, Vol. 43, No. 6, 1997, pp. 1605-1610.

${ }^{20}$ Raman, V., Pitsch, H., and Fox, R. O., "Hybrid large-eddy simulation/Lagrangian filtered-density-function approach for simulating turbulent combustion," Combustion and Flame, Vol. 143, 2005, pp. 56-78.

${ }^{21}$ Reid, R., Prausnitz, J., and Polling, B., "Molecular Theory of Gases and Liquids," 1987.

${ }^{22}$ Franzelli, B., Riber, E., Gicquel, L. Y. M., and Poinsot, T., "Large Eddy Simulation of combustion instabilities in a lean partially premixed swirled flame," Combustion and Flame, Vol. 159, No. 2, 2012, pp. 621-637.

${ }^{23}$ Mayer, W., Telaar, J., Branam, R., Schneider, G., and Hussong, J., "Raman measurements of cryogenic injection at supercritical pressure," Heat and Mass Transfer/Waerme- und Stoffuebertragung, Vol. 39, No. 8-9, 2003, pp. 709-719.

${ }^{24}$ Yoo, J. Y., "The turbulent flows of supercritical fluids with heat transfer," Annual Review of Fluid Mechanics, Vol. 45, No. 1, 2013, pp. 495-525.

${ }^{25}$ Panchapakesan, N. and Lumley, J., "Turbulence measurements in axisymmetric jets of air and helium. Part 1. Air jet," Journal of Fluid Mechanics, Vol. 246, 1993, pp. 197-223.

${ }^{26}$ Boersma, B., Brethouwer, G., and Nieuwstadt, F., "A numerical investigation on the effect of the inflow conditions on the self-similar region of a round jet," Physics of fluids, Vol. 10, No. 4, 1998, pp. 899-909.

${ }^{27}$ Pitsch, H., Sejser, R., and Varatharajan, B., "A Guide to FlameMaster," .

${ }^{28}$ Peters, N., "Laminar diffusion flamelet models in non-premixed turbulent combustion," Progress in energy and combustion science, Vol. 10, No. 3, 1984, pp. 319-339.

${ }^{29}$ Bilger, R., Stårner, S., and Kee, R., "On reduced mechanisms for methane air combustion in nonpremixed flames," Combustion and Flame, Vol. 80, No. 2, 1990, pp. 135-149.

${ }^{30} \mathrm{Kim}, \mathrm{J}$. and Williams, F., "Structures of flow and mixture-fraction fields for counterflow diffusion flames with small stoichiometric mixture fractions," SIAM Journal on Applied Mathematics, Vol. 53, No. 6, 1993, pp. 1551-1566. 